\title{
De la Bibliotecología al Sistema de Conocimientos Científicos Bibliológico-Informativo
}

Emilio Setién Quesada

Inves ti ga dor Ti tu lar, Jefe del De par ta men to deInvestigaciones de laBibliotecaNacional de Cuba

Salvador Gorbea Portal

Investigador Titular visitante del Centro Universitario de Investigaciones Biblio te co ló gi cas de la Universidad Nacional Autó no ma de México.

\begin{abstract}
RESUMEN
$S$ e presenta, a par tir de tres presu pues tos car di na les y con un en fo que in ter dis ci plina rio, un mo de lo teóricosobre el Sistemade Conocimientos CientíficosBibliológico-Informativo, en el que se de finen las dis ci pli nas cien tífi cas que lo in te gran, asícomo las re la cio nes in ter dis ci pli na rias que se dan en tre és tas. Se des ta can, tam bién, aque llas dis ci pli nas que ac túan como rec to ras con re la ción al surgi mien to del res to de las que com po nen el mo de lo y el ni vel de in te gra ción con el que de be ana lizar este Sistema de Conocimientos.
\end{abstract}

\section{ABSTRACT}

$\boldsymbol{A}$ theoreticalmodelconcerning the System of Scientific-Librarian-Infor mative Knowledge will be presented based on three cardinal presuppositions and with an interdiscplinary focus in which the scien ti fic dis ci pli nes that con form it will be de fi ned, as well as the in ter dis ci pli nary re la tions which occur among them. Also stressed will be tho se dis ci pli nes which act as rec tors in re la tionwith the deve lopment of the other dis ci pli nes which form the mo del, and the le vel of in te rac tion with which this Knowledge System should be analyzed.
$D$ acuerdo con criterios muy generalizados actualmente en el mundo, se considera que las Ciencias de la Información incluyen a la Archivología, la Bibliografía o Bibliografología, la Bibliotecología y la Documentación o Ciencia de la Información, propiamentedicha.

Sin embargo, algunos autores opinan que la denominación de Ciencias de la Información pue de im pli car la in clu sión del Pe rio dis moen el con jun to de las dis ci pli nas an tes men ciona das. Es necesario señalar al respecto que mien tras que la Archi vología, la Bibliografolo gía, la Bi blio te co lo gía y la Cien cia de la Información se ocu pan de la in for ma ción y sus portadores, el Periodismo se ocupa esencialmen te de trans for marhe chos en in for ma ción. Parasus traerse a es tas dis qui si cio nes, en este trabajo se ha op ta do porde no mi nar a ese conjunto de disciplinas "disciplinas Bibliológico-Informativas", en tanto, A. Sokolov las clasifica como disciplinas que pertenecen al complejo bibliológico de las ciencias comunicativasinformativas. ${ }^{(1)}$
He cha esta sal ve dad, el tra ba jo que aho ra se ofrece a la con si de ra ción del lec tor in cursiona en el es tu dio de las re la ciones me to do ló gi cas que existen entre las disciplinas bibliológico-in for ma ti vas, y en tre ellas y otras que, sin pertenecer al complejo bibliológico identificado por Sokolov, resultan rectoras para su existencia y desarrollo, como por ejemplo la His to ria y la Me to do lo gía de la In ves ti ga ción.

Para la incursión antes señalada se parte de tres presupuestos cardinales:

a) Elde sa rrollo de las dis ci pli nas biblio lógi co-in for ma tivas se produce a partir de dos movimientos convergentes:

- el que va desde la creación, producción y difusión de documentos, hasta el uso que se hace de sus contenidos como resultado de la actividad informativa de los individuos. Definido éste por A.I.Mijailov en su obra(2) como el ci clo de las ta reas y eta pas de las actividades de información científica(Creación-Recopilación-Procesa- miento Analítico Sintético-Almacenamiento-Búsqueda-Difusión-Uso de la Información).

- El que par te de la ac ti vi dad in for ma tiva de los individuos y el uso de la infor ma ción y se di ri ge hacia la difu sión, produc ción y crea ción de do cu men tos y de la información misma, aspecto que E. Bue no ${ }^{(3)}$ en mar ca den tro de la Metodología de la Investigación en los Sistemas de Conocimientos, uno de los tres componentes principales del proce so de co no ci mien to, y que se manifiesta al añadir a estos sistemas nuevosconocimien tos e in for mación.

b) La Archivo logía, la Bi blio gra fo lo gía y la Biblio te cología se en marcan en el primer movimiento y como disciplinasrectoras, la His to ria se co rres pon de con la pri me ra y la Bibliología con las segundas; la Ciencia de la Información pertenece al segundo movimiento y se apoya como disciplina rec to ra en la Me to do lo gía de la Investigación. 
c) Es en la especialización de Referencia, propia de la Bibliotecología, donde convergen am bos movimien tos.

Se asumen, asimismo, las definiciones siguientes:

La Bibliología es, para algunos autores, la ciencia más general que abarca todo el comple jo bi blio ló gi co de las cien cias co mu ni ca ti vo-informativas. Es tu dia al li bro y al sis te ma del libro en los planos históricos, actual y de pronóstico. Comprende la teoría, la historia, metodología y organización de la actividad edi to rial, del di se ño del li bro, de la bi blio tecnia, del co mer cio del li bro, de la ac ti vi dad bibliográfica, de la actividad bibliotecaria, de las es ta dís ti cas de las pu bli ca cio nes y del uso social de las obras manuscritas e impresas, disciplinarec to ra que pro pi cia el surgi mien to de la Bi blio gra fo lo gía y la Biblio te co lo gía y que, al decir de O. Chubarian, enriquece la base teórica de la Bibliotecología $a^{(4)}$.

La Bi blio gra fo lo gía, por su par te, es la dis cipli nade las cien cias so ciales, per te ne cien te al complejo bibliológico de las ciencias comunicativo-informativas, que es tu dia a la activi dad bibliográfica, esto es, a los procesos de crea ción, di fu sión y uso de las com pi la cio nes bibliográficas ${ }^{(5)}$.

La Biblio te co lo gía es la dis ci pli na de las ciencias so cia les, per te ne cien tes tam bién al comple jo biblio ló gi co de las cien cias co mu ni ca ti vo-informativas, que estudia la actividad biblio te caria. Laactividadbibliotecariacompren de, a gran des ras gos, los pro ce sos de formación, acumulación y uso del fon dobibliotecario, así como los métodos, técnicas y recursos que se uti li zan en ellos, en sus re laciones con el mediosocial ${ }^{(4)}$ (figura 1)

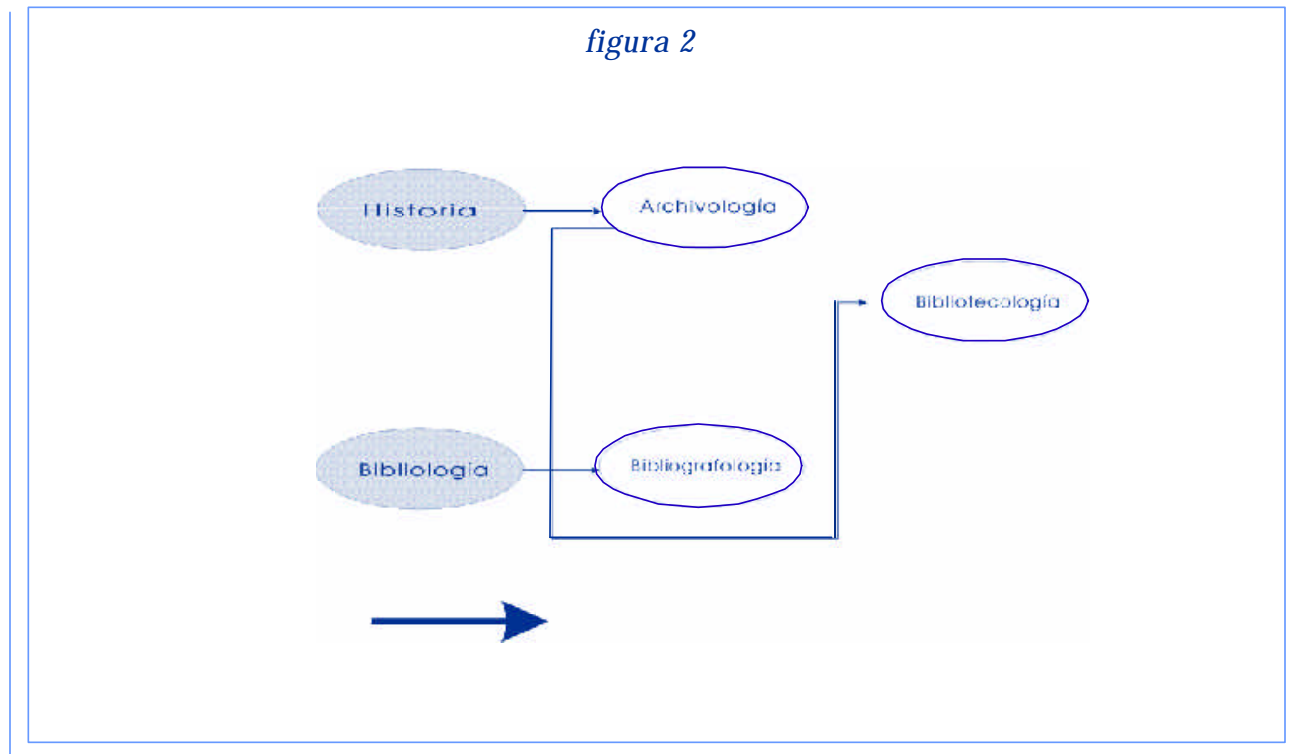

La Archivología es una disciplina que se desarrolla en la zona de interpenetración de las ciencias históricas y las disciplinas del comple jo biblio ló gi co de las cien cias co mu ni ca tivo informativas.

Estudia la actividad archivística, especificamen te los proble mas teó ri cos, his tóricos, metodológicos y organizativos referentes a los documentos y fondos archivísticos $\left({ }^{(6)}\right.$, así como la apli ca ción de la Pa leon to lo gía, la Diplomática, la Onomástica, la Toponimia y la Cro no lo gía al tra ta mien to de los documen tos que le son pro pios (figura 2).

La Ciencia de la Información es una ciencia Interdisciplinariaporque se for ma en la zona de interpenetración de varias ciencias, entre las que ocupan un lugar importante la Metodología de la Investigación Científica y las disciplinas bibliológico-informativas clásicas (Archivología, Bibliografología y Biblio-

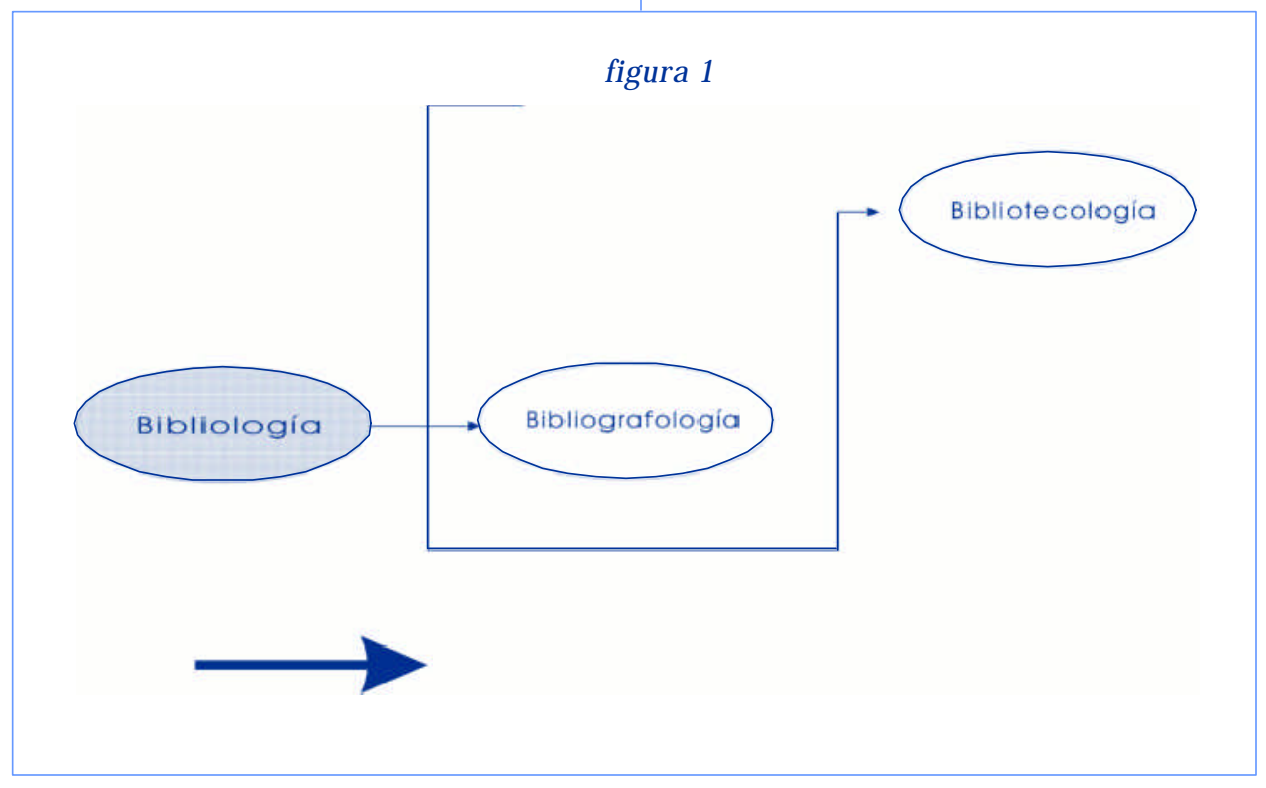

tecología). Así considerada, se incluye también en el complejo bibliológico de las disciplinascomunicativo-informativas.

La Ciencia de la Información estudia la actividadcientífico in for mativaen su conjun toy, muy especialmente, las propiedades, estructu ra y re gu la ri da des de la in for ma ción cien tí fica, para lo que se apoya en conocimientos sobre métodos heurísticos, y en los desarrolla dos por la Bi blio lo gía en su con jun to, bá sica men te por la Biblio gra fo lo gía y la Bi blio tecología. Comprende, además, el estudio de los proble mas re la ti vos a la in for ma ción desde el puntode vistalingüístico, psicológico, sociológico, matemático, cibernéticoy tecnológico(7).

Para el es tu dio de la es truc tu ra, las pro pie dades y las regularidades de la información científica, la Ciencia de la Información se apo ya en los cuer pos teó rí cos de va rias dis ciplinas, como quedó apuntado de su definición, in clui dos los apor tes a la Bi blio lo gía, la Bibliografología y la Bibliotecología.

Lasdisciplinas, especialidades y especializaciones con te ni das en las de fi ni ciones an te riores ha cen evi den te las re la cio nes de las dis ciplinasbibliológico-informativascondiversas ra mas de las Cien cias Téc ni cas y de las Ciencias So ciales, que con tribu yan a su de sa rrollo más profun do y mul ti la te ral. En tre esas dis ciplinas se des ta can la Biblio logía, la His to ria y la Me to do lo gía de la In ves ti ga ción como secto res del co no ci mien to que son rec to res de las disciplinas bibliológico-informativas en tanto de fi nen la cua li dad de los pro duc tos que se logran por las actividades estudiadas por esa disciplinas, a saber: la Actividad Bibliotecaria, la Bibliográfica, la Archivística, y la Científico-Informativa. 
Seencuen tran asi mis mo las que tie nencarácter complementario: la sociología, la psicología, la pedagogía, la economía, la ma te má tica, la tec no logía, la lin güís tica, laci bernética, en tre otras.

La Referencia es una especialización de la rama de la Biblio te co logía de no mi na da "Trabajo con los lectores". La Referenciaestudia los problemas relativos a la orientación del lec tor, la en tre ga de in for ma ción bi blio gráfi ca, la eva cua ción de con sul tas, la in for ma ción bi blio te ca ria y las téc ni cas de in for ma ción.

Para el es tu dio de los proble mas re la ti vos a la información bibliográfica, la Referencia se apo ya en los co no ci mien tos de sa rrolla dos por la Bibliografo lo gía so bre mé to dos y compilacio nes bi blio gráfi cas, y para elde las téc ni cas de información en los desarrollados por la Cien cia de la In for ma ción so bre las pro pie dades y es truc tu ra de la in for ma ción cien tífica y su procesamiento analítico sintético: métodos que tie nen su ori gen en la Me to do lo gía de laInvestigación Científica (figura 3 ).

Re la ciones si mi la res se produ cen en tre la Biblio gra fo lo gía y la rama de la Bi blio te co lo gía de di ca da a los ca tálo gos bi blio te carios, puesto que esta es pecia li dad de la Bi blio te co logía se ocu pa de la adap ta ción de los mé to dos biblio grá fi cos al tra ba jo de las bi blio te cas, donde los do cu men tos no sólo se des criben y clasifican sino que se acumulan y se recuperan para su uso.

La historia de las bibliotecas (Bibliotecografía), por su parte, estáes tre cha men te re la ciona da con la his to ria de la es cri tu ra y el li bro y los fon dos bi blio te ca rios con los as pec tos correspondientes a las ramas de la Bibliología que estudian la actividad editorial, la Bibliotec nia y el co mer cio del li bro, por lo que la Bibliotecología se vincula estrechamente con los estudios del complejo bibliológico de las disciplinascomunicativo-informativasrelativas a los pro ce sos de crea ción, di fu sión y utilización de las obras manuscritas impresas.

La Archivología estudia métodos y técnicas surgidos en la esfera de la Bibliografología para su aplicación en los archivos. También es tu dia proble mas de al ma ce na mien to y protección de fondos y servicios de referencia cer ca nos a los que es tu dia la Biblio te co lo gía (figura 4).

En con jun to, las re la cio nes has ta aquí des critas se pueden in te grar en la figura 5.

O de for ma más sin té ti ca y con cep tual en la figura 6 .

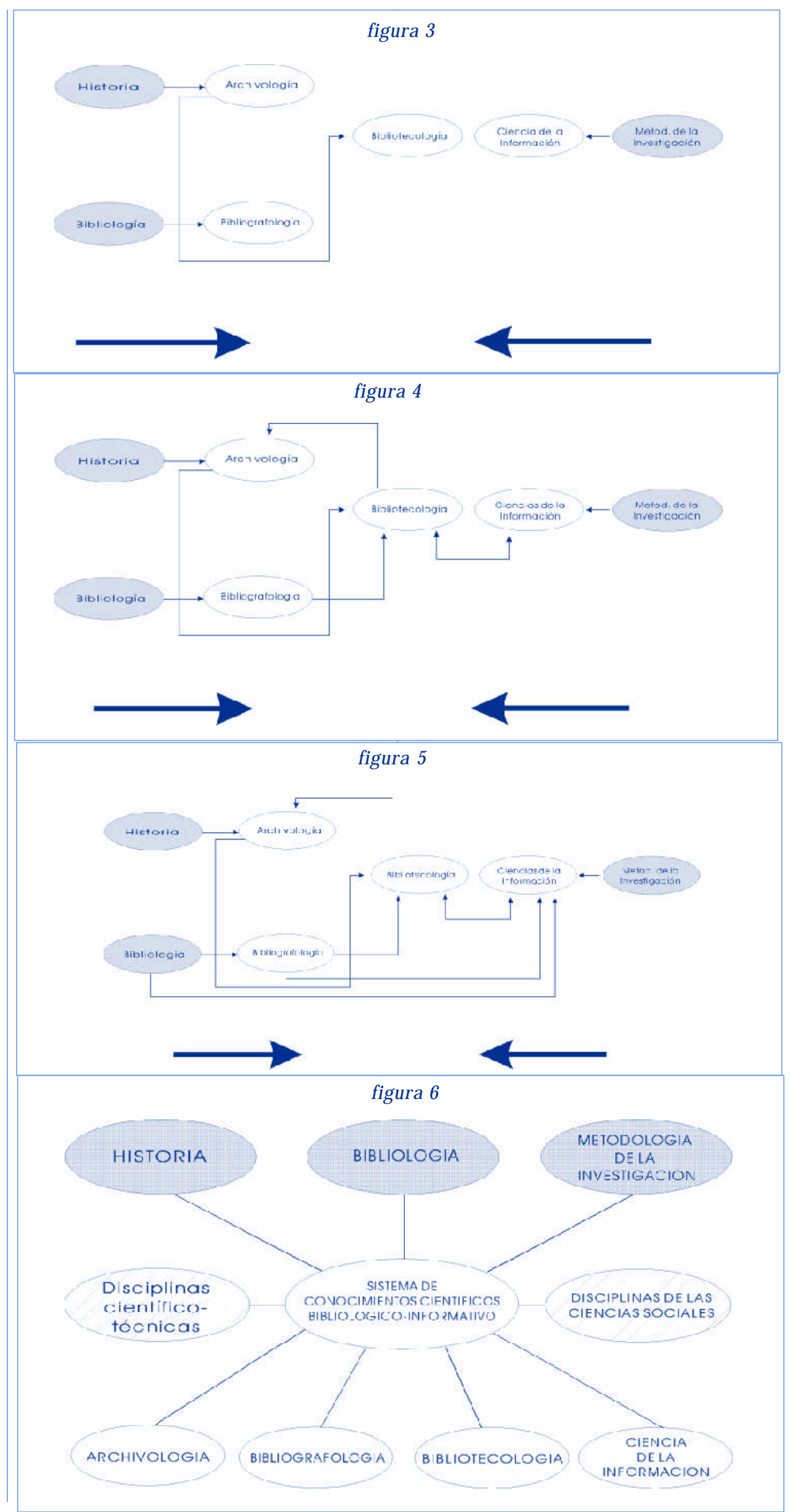


No sería ocioso señalar que las relaciones multidisciplinarias, es decir, las que guardan re la ción con la di ver si dad de fuen tes de co nocimientos sobre el tema - hasta aquí representadas mediante el uso de los esquemas mos tra dos para la fun da men ta ción de la existen cia de un sis te ma de co no ci mien tos cien tíficos bibliológico-informativo- no deben ser in ter pre ta dos de for ma ab so lu ta ni mu cho menosais lados; dicharepresentación res ponde a un mo de lo o es que ma que pre ten de ayudar a com pren der de for ma di dác ti ca la presenciadelreferidosis te ma deconocimien tos, así como los ele men tos (dis ci pli nas, es pe cialidades y es pecializaciones) que lo in te gran y las re la cio nes que se dan en tre ellas.

Sin em bar go, la na tu ra le za de un ob je to de estu dio tan di ver so y com ple jo como lo es la infor ma ción, re quie re no sólo de un análi sis aislado desde la actividad o perspectiva Archivística, Bibliotecaria, Bibliográfica, o Científico-Informativa.

En este sen ti do se re quie re ade más de un enfoque integral en el que confluya la aplicación de una gama va ria da de mé to dos y téc nicas, pertenecientes a varias disciplinas que propicien la solución de un problema de forma in ter dis ci pli na ria, es de cir, la que nos lleva al pensamiento de que es ta mos ante un te- rreno donde interactúan múltiples dimensiones de un mismo obje to de es tu dio.

\section{CONSIDERACIONES FINALES}

A menudo se contempla en Instituciones Infor mativaslanecesidad deorganizarsistemas de in for ma ción en los que sub sis ten va rias de estasactividades para dar solución, de manera integral, a un problema informativo de la práctica social.

De igual ma ne ra ocu rre en la do cen cia y la inves tigación de estesis te ma de conoci mien tos, en el que la formación de un especialista o la solución de un problema requieren también de un enfoque interdisciplinario, si se trata entonces de resolver un problema (docente o de in ves ti ga ción) no des de la pers pec ti va Archivística, Bibliotecaria, Bibliográfica oTecnológica, o sea, de forma multidisciplinaria, sino de re solver lo des de la pers pec ti va onaturaleza del objeto de estudio y los problemas asociados a éste.

Final men te, debe con si de rar se que el bi blio teca rio, el ar chi vis ta, el bi blió gra fo y el in for ma dor es tán obli ga dos a co no cer el sis te ma de conocimientos bibliológico-informativos en su conjunto. Sin embargo, ellos no necesariamente tienen que saber y saber aplicar todos esos co no ci mien tos, sino sólo aque llos que co- rrespondan a su esfera de acción o actividad (bibliotecaria, bibliográfica, archivística o cien tífico-in for mativa) obien, en susacti vidades como cien tífi cos y docen tes que se de senvuelven en te mas y disciplinasespecíficas.

En la actualidad el volumen de los conocimientos acumulados por cada disciplina bibliológico-informativa, asícomolaespecificidad de los modos que cada una de las ac ti vi da des co rres pon dien tes em plea para obte ner los pro duc tos que le son pro pios (com pilaciones bibliográficas, fondo bibliotecario, fondoarchivístico, información lógica), impone un des pla za mien to en lo que debe co no cer, sa ber y sa ber apli car un pro fe sional.

En la esfera del trabajo bibliotecario, por ejemplo, el profesional de pregrado puede for mar se de acuer do con los cri te rios ex puestos en el párrafo anterior, pero es necesario formar especialistas de postgrado, al menos para las áreas de for ma ción de fon dos, procesamiento, trabajo con los lectores, administra ción y con ser va ción, en tre otras; lo que implica no sólo saber el conjunto de conocimientos que corresponden a la Bibliotecología en general, sino el saber y saber aplicar aquellos que correspondan a la especialidad o especialización de ésta donde se desenvuelvaelindividuo.

\section{REFERENCIAS}

1 SOKOLOV, A.U. Sistemadecienciascomunicativo-informativas. Op. Cit. en Bi bliografía, p.5-15

2 MIJAILOV, A.I. y R.S. Gui la rie vskii.CursoIntro suctoriode Informática/Documen tación. Op. Cit. en Bibliografía, p. 190

3 BUENOSANCHEZ, Era mis.Notas sobre Me to dologíadela Investigación. Op. Cit. en Bibliografía, p. 9-12

4 Chubarian, O.S. Bibliotecología General. Op. Cit. en Bibliografía, p. 53-54

- ABRAMOV, K.I.Bibliotekovedenie:ObschiiKurs.Op. Cit.en Bibliografía, p. 27-62

- SETIEN, Emi lio y Sal va dor Gor bea. "Con cep tos métri cos de las dis ci pli nas Biblio teco-informativas”. Op. Cit. en Bibliografía, p.3-17

5 KORSHUNOV, O.P.Bibliografiya:ObschiiKurs. Op. Cit. en Bibliografía. p. 204-210

- PrimerSimposiode Bibliografía. In for me Fi nal.Op. Cit. en Bibliografía, p. 75-78

- SETIEN, Emi lio y Sal va dor Gor bea. Concep tos métricos de las disciplinas Biblioteco-Informativas. Op. Cit. en Bibliografía, p. 3-17 
VILA, TA NIA. "Selección, preparación profesional y elevación del nivelideológico de los trabajadoresde Archivos". Op. Cit. en Bibliografía, p. 95-134

- CURRAS, Emi lia.Lasciencias deladocumentación. Bibliotecología, Archivología, Documentacióne Información. Op. Cit. en Bi bliogra fía, p. 21

7 SETIEN, Emi lio y Sal va dor Gor bea. "Con cep tos mé tri cos de las dis ci pli nas Bi blio teco-Informativas." Op. Cit. en Bibliografía,p. 3-17

\section{BIBLIOGRAFIA}

ABRAMOV, K.I. Bibliotecokovedanie: ObschiiKuvs.- Moskva: Knizhnaja $\mathrm{Pa}$ la ta, 1988.-224 p. (En ruso)

BUENO SANCHEZ, Eramis. Notas sobre Metodología de la Investigación._La Ha ba na : CE DEM,1993.—130 p. (Por pu bli car)

BUONOCORE, Do min go. DiccionariodeBibliotecología; términos relativos a la bibliología, bibliografía, bibliofilia, biblioteconomía, archivología, documentología, tipografía y materias afines. Buenos Aires: Marymar, 1976. $465 \mathrm{p}$.

CARriongutierrez, Ma nuel.- Ma nual de Bi blio te cas.-Ma drid : Pi rámide, 1988 . $-756 \mathrm{p}$.

CURRAS, Emi lia. Las cien cias de la do cumen ta ción. Biblio te co lo gía, Archivología, Documentación e Información.- Barcelona: Mitre, 1982. $240 \mathrm{p}$.

CHUBARIAN, O.S. BibliotecologíaGeneral/trad. An to nia Tris tá Pé rez.- La Habana: Edi to rial Cien tífico-Téc nica, 1981.—367 p.

KORCHUNOV, O.P. Bibliografiya: Obschii Kuvs.- Moskva: Kniga, 1981.- 511 p. (En ruso)

MIJAILOV, A.I. y R.S. Gui la rie vskii.CursoIntroductoriodeInformática/Documenmtaria. - Caracas: Fundación Instituto Venezolano de la Productividad, 1974. $236 \mathrm{p}$.

Primer Simpo sio de Bibliografía. In for me Fi nal. BIBLIOTECAS (La Ha ba na) 29(1-4):75-78, enero-diciembre, 1991

SEQUEIRAORTIZ, De ya ni ra y Zai da Se quei ra Or tíz.La Biblio te cologíacomo ciencia.-San José, Costa Rica: Publitero, 1988. 218 p.

SETIEN, Emilio y Salvador Gorbea. "Conceptos métricos de las disciplinas Biblioteco-Informativas."-ActualidadesdelaInformación Científica y Técnica (La Habana) 21(156):3-17, 1990.

SOKOLOV, A.U. "Sistema de ciencias comunicativo-informativa". Actualidades dela Información Científica y Téc nica(La Ha ba na)3(128):5-15, 1986

VILA, Ta nia. "Se lec ción, pre pa ra ción pro fe sio nal y ele va ción del ni velideoló gi co de los tra ba ja do res de Ar chi vos". En: VII Confe ren cia de di recto res de Ar chi vos Cen tra les de los Partidos Co mu nis tas y Obre ros de paises socialistas. Memorias. - La Habana: Instituto de Historia de Cuba, 1985. 552 p. 